\title{
Abstracts presented at the South West ENT Academic Meeting 2017, Bath, UK
}

\author{
Management of patients with unilateral facial nerve palsy \\ in the acute setting at Royal United Hospital, Bath \\ V Evans, A Henderson, S Gillett \\ From the Royal United Hospital, Bath

\section{Introduction} \\ The inability to move facial muscles caused by unilateral \\ lower motor neuron facial nerve palsy can have both a sig- \\ nificant physical and psychological impact. Acute presenta- \\ tion may be to one of several different medical specialties.
}

\section{Objectives}

We aimed to: assess the quality of investigation and management of patients with acute lower motor neuron facial nerve palsy, compare with current national guidance, and determine whether ENT assessment changes the outcome.

\section{Methods}

A retrospective notes analysis was conducted of all patients presenting to the emergency department, medical assessment unit and medical ambulatory care with unilateral lower motor neuron facial nerve palsy during a six-month period (1 September 2016 to 1 March 2017). Parameters of measurement included: initial ENT assessment, clinical examination, further imaging, administration of steroids, antivirals and eye protection, and appropriate follow up.

\section{Results}

Of a total of 17 patients, only 5 were seen by ENT. Of those, 100 per cent underwent otoscopy and 80 per cent parotid examination, compared with 0 per cent and 8.33 per cent respectively for the remaining cohort. Of the total patients, 64.7 per cent underwent imaging (most commonly computed tomography of the head -47.1 per cent). Eighty-two per cent of patients received steroids appropriately, within 72 hours in all cases. Thirty-five per cent of patients received acyclovir (all within 72 hours), but only one patient was examined with otoscopy (by ENT). One hundred per cent of those patients seen by ENT were given eye protection, compared with 58.3 per cent of the remaining patients. Eighty per cent of those seen by ENT were followed up in the outpatient department, compared with 25 per cent of the remainder.

\section{Conclusion}

A significant variance in examination and management is evident. It is therefore clear that further education is essential for non-ENT specialties encountering patients with facial nerve palsy. A diagnostic and management tool has been developed.

Complete audit cycle of otitis externa in casualty clinic after otitis externa pathway introduction

K Davies, F Gregory, D Collis, H Elhassan

From the Morriston Hospital, Swansea, Wales

\begin{abstract}
Introduction
Otitis externa is common; 10 per cent of the UK population will be affected in their lifetime, and the yearly incidence is 1 per cent. Most cases are managed in primary care; however, otitis externa referrals represent a large proportion of cases seen by ENT junior doctors in the casualty clinic.
\end{abstract}

\section{Objective}

We aimed to introduce a more efficient otitis externa pathway, advising senior review or referral to the outpatient department after the second follow-up appointment, thereby reducing unnecessary casualty clinic appointments.

\section{Methods}

An online casualty clinic diary was searched for appointment frequency of all otitis externa patients between August 2015 and December 2015. Departmental guidelines were introduced and training was provided to junior doctors. A second prospective four-month audit cycle was performed.

\section{Results}

From August to December 2015, there were 138 otitis externa referrals; 84 patients (61 per cent) were discharged after the first casualty clinic appointment and 54 patients (39 per cent) required follow up. Of those followed up, 46 patients (85 per cent) were discharged after 1 or 2 followup appointments, and 8 patients ( 15 per cent) were followed up on 3 or more occasions. After pathway implementation (December 2015 to March 2016), 66 of the 81 patients (81 per cent) required no follow up, and 100 per cent of those who were followed up were brought back to the casualty clinic on 2 or fewer occasions.

\section{Conclusion}

The new otitis externa pathway increased the number of discharges after the first visit by 33 per cent. The number of first follow-up appointments was reduced by 50 per cent and second follow-up appointments by 31 per cent. Following its introduction, the pathway was adhered to in 100 per cent of cases.

A case of cerebral venous sinus thrombosis and profound bilateral sensorineural hearing loss following multiple presentations with relapsing polychondritis

M E Hopkins, A S Harris, J Clarke

From the Royal Gwent Hospital, Newport, Wales

\section{Introduction}

Relapsing polychondritis is a rare autoimmune condition resulting in severe, progressive inflammation of cartilaginous tissue. Previous case reports have established a connection with profound sensorineural hearing loss (SNHL). Here, we present the case of a patient who suffered from sigmoid sinus and internal jugular vein thrombosis, and subsequently 
experienced profound bilateral SNHL secondary to relapsing polychondritis.

\section{Case report}

The patient, with a background of polymyalgia rheumatica and rheumatoid arthritis, initially presented to ENT with symptoms of perichondritis, fever and local inflammation of the pinna. She was treated with intravenous antibiotics and discharged. Three months later, she presented with inflammation of the opposite pinna. In addition, there was evidence of rotational vertigo and bilateral tinnitus. She was discharged following a course of intravenous antibiotics, despite little improvement in inflammatory markers. She was admitted again one week later with profound bilateral SNHL. Auditory brainstem response testing confirmed bilateral 'dead ears'. A contrast computed tomography (CT) scan demonstrated sigmoid sinus thrombosis with extension into the internal jugular vein. She was anticoagulated with warfarin and the underlying condition was treated with methylprednisolone. Thus far, her hearing has showed no signs of improvement and she is awaiting unilateral cochlear implantation.

\section{Discussion}

We postulate that the recognised complication of bilateral SNHL in a patient with relapsing polychondritis is secondary to underlying cerebral sinus thrombosis and its locally neurotoxic effects. This case highlights the importance of early CT scanning and pure tone audiograms in cases of suspected relapsing polychondritis, to allow for prompt intervention, with the aim of preventing profound hearing loss in this cohort of patients.

Scoping: a revolution in middle-ear surgery. Trainees' perspectives on endoscopic ear surgery

E K Bhargava, B Wong, P Coyle, A Patel, C Swords, A Qayyum

From the Peterborough City Hospital

\section{Introduction}

Minimally invasive endoscopic ear surgery is rapidly emerging as a mainstream surgical modality, with recent literature highlighting its advantages over traditional approaches. As more surgeons are adopting the technique, trainees in otolaryngology are at the midst of what some may term as a 'revolution' in middle-ear surgery.

\section{Objective}

This study aimed to highlight trainees' beliefs and concerns regarding this technique via a cross-sectional survey of British otolaryngology trainees through an online survey.

\section{Methods}

A cross-sectional survey was conducted of British otolaryngology trainees between December 2016 and March 2017 via an online survey comprising 13 questions.

\section{Results}

Sixty per cent of responding trainees used endoscopes in otology practice; however, none used endoscopes as the primary instrument for cholesteatoma surgical procedures. A superior view, less post-operative morbidity and better cosmesis were the primary perceived advantages, whilst one-handed surgery was the most challenging perceived limitation. Eighty-five per cent of trainees felt there was a need for specific training in endoscopic techniques, and the majority of trainees agreed that they would use endoscopes in the foreseeable future.

\section{Conclusion}

This study relates today's trainees' opinions regarding endoscopic ear surgery, considered a 'revolution' in otology by some surgeons. It provides keen insight into their reservations regarding the procedure, and their suggestions for incorporating it into formal otolaryngology training.

\section{Pain following ear surgery}

E R Taggert ${ }^{1}$, A Jardine ${ }^{2}$

From the ${ }^{1}$ University of Bristol and ${ }^{2}$ Royal United Hospital, Bath

\section{Introduction}

Pain following ear surgery is rarely a prominent complication and little is written about it. However, some patients (nine known to the senior author) have severe persistent pain.

\section{Objectives}

The study aimed to assess the pain scores following ear surgery in a cohort of patients having mastoid surgery and to review the nine patients with severe persisting pain.

\section{Methods}

Informed consent was obtained. Any history of previous or current chronic pain was established. Patients scored their overall pain (from 1 to 10) in the first 24 hours following surgery and on the 4 th post-operative day.

\section{Results}

Nineteen patients were included in the prospective audit. The median pain score of 15 patients with no history of chronic pain was 2.9 at day 1 and 1.5 at day 5 . The median pain score for 4 patients with chronic pain (not in the ear) was 6.25 at day 1 and 7.3 at day 5. A retrospective analysis was conducted of the 9 patients with severe pain following ear surgery; 8 were female and all were under 30 years of age. Ear pain prior to surgery was present in three patients. All reported high levels of pain in the first 24 hours that persisted.

\section{Conclusion}

Pain following ear surgery can be severe and persistent in a small number of individuals. Predictors of pain following ear surgery include pre-operative ear pain, chronic pain, younger age and female sex. In light of the Montgomery ruling, pain needs to be included in the discussion prior to ear surgery, and knowledge of historical factors likely to predict pain need to be considered.

How sensitive is pre-operative fine needle aspiration cytology in the diagnosis of a parotid mass? A review of 50 consecutive parotidectomies at the Norfolk and Norwich Hospital

R Jackson, S Burrows

From the Norfolk and Norwich University Hospital, Norwich

\section{Introduction}

The principle investigation of choice for a parotid mass is an ultrasound scan with fine needle aspiration cytology (FNAC). The FNAC results influence whether further investigations are conducted and indicate the need for operative intervention. 


\section{Objectives}

We aimed to audit how patients presenting with a parotid mass were pre-operatively investigated, and to compare the sensitivity of pre-operative FNAC with post-operative histology.

\section{Methods}

A retrospective review of the last 50 parotidectomies performed in our institution was undertaken. Pre-operative FNAC and core biopsy results were compared to post-operative histology.

\section{Results}

All 50 patients had a pre-operative ultrasound scan with FNAC. Two patients (4 per cent) had a repeat FNAC and seven (14 per cent) had core biopsies prior to parotidectomy. Thirty-seven patients (74 per cent) were given a single diagnosis on initial FNAC, six patients (12 per cent) had multiple diagnoses on FNAC and seven patients (14 per cent) had no clear diagnoses indicated by initial FNAC results. In 17 patients ( 34 per cent), the initial FNAC result did not match post-operative histology. More importantly, four patients ( 8 per cent) had a histological diagnosis of malignancy despite pre-operative investigations showing benign disease. Four patients ( 8 per cent) had concurrent neck dissections alongside a parotidectomy. Histological findings included: pleomorphic adenoma (48 per cent), Warthin's tumour (26 per cent), carcinoma ex-pleomorphic adenoma (10 per cent) and eight other parotid lesion subtypes.

\section{Conclusion}

Sixty-six per cent of patients had an FNAC result that matched the post-operative histological findings, highlighting the importance for clinicians not to rely solely on FNAC for diagnosis. Repeat FNAC and core biopsies can be used to further investigate a parotid mass, with surgical excision the 'gold standard' if diagnosis remains unclear.

\section{A report on 15 years of clinical negligence claims in rhinology}

T Geyton, T Odutoye, R Mathew

From the Royal United Hospital, Bath

\section{Introduction}

During 2010-2011, the National Health Service (NHS) litigation bill surpassed one billion pounds sterling (GBP) for the first time. Systematic analysis of malpractice complaints allows for the identification of errors, and can thereby improve patient safety and reduce the burden of litigation claims on health services.

\section{Objective}

This study was designed to determine the characteristics of medical negligence claims in rhinology.

\section{Methods}

Claims relating to ENT between 1995 and 2010 were obtained from the NHS Litigation Authority and analysed.

\section{Results}

The series contains 65 closed claims that resulted in payment totalling 3.1 million GBP. Fifty claims were related to surgical complications. Functional endoscopic sinus surgery and septoplasty were the procedures most commonly associated with successful claims. There were 11 cases of orbital injury, including 6 cases of visual loss and 5 cases of diplopia. The most common cause of a claim was failure to recognise the complication or manage it appropriately. Lack of informed consent was claimed in eight cases. Other claims arose because of: delayed surgery (one), and errors in out-patient procedures (two), diagnosis (six) and medical management (three).

\section{Conclusion}

Claims in rhinology are associated with a high success rate. Careful investigation of patients and ensuring adequate informed consent can reduce litigation. Where there is a suspicion of orbital damage, early recognition and intervention is needed to reduce long-term injury to the patient.

\section{Primary endoscopic treatment of cholesteatoma - a realistic alternative? \\ C Hunter ${ }^{1}$, S Broomfield ${ }^{2}$, P Robinson ${ }^{2}$, D Hajioff ${ }^{2}$,}

From the ${ }^{1}$ University of Bristol and ${ }^{2}$ University Hospitals Bristol NHS Foundation Trust

\section{Introduction}

Recently, there has been increased interest in using the endoscope for cholesteatoma surgery, as the endoscope allows access to areas of the middle ear that are otherwise impossible to visualise. Some advocate use of the endoscope as the primary tool in cholesteatoma surgery, whilst others use it as an adjunct to the microscope. Whilst there is no clear consensus, the posterior edge of the lateral semicircular canal is often taken to be the limit of accessibility using the endoscope for disease that extends into the mastoid.

\section{Objective}

This study aimed to identify the proportion of cholesteatoma cases performed using the endoscope that might have been possible endoscopically.

\section{Methods}

A retrospective analysis was performed of 142 consecutive mastoid operations for cholesteatoma (133 combined approach tympanoplasties and 9 atticotomies). The extent of disease was graded by the operating surgeon using the attic-tympanum-mastoid ('ATM') system. A judgement regarding suitability for endoscopic access was made.

\section{Results}

It was judged that all 9 atticotomies (100 per cent) and 65 combined approach tympanoplasties (49 per cent) would have been possible using a purely endoscopic technique. Sixty-eight combined approach tympanoplasties (51 per cent) would not have been possible endoscopically given the extent of mastoid disease and, in one case, a very narrow external auditory canal.

\section{Conclusion}

In our practice, the extent of cholesteatoma encountered means that the endoscope is unlikely to replace the microscope completely. However, a significant proportion of mastoid procedures may be possible endoscopically, with potential for reduced morbidity. 
The histological yield of $\mathbf{5 0}$ consecutive patients undergoing hemi-thyroidectomy for Thy-3 and Thy-4 lesions at the Norfolk and Norwich University Hospital

\section{R Jackson, S Burrows}

From the Norfolk and Norwich University Hospital, Norwich

\section{Introduction}

Thyroid lumps are common within the general population, with most going completely undetected. Patients with lumps who are referred to the ENT clinic are often investigated with an ultrasound scan. Those with indeterminate or abnormal lesions on ultrasound should undergo fine needle aspiration cytology (FNAC). 'Thy' and ultrasound 'U' classifications have been established to help determine features of malignancy. Hemi-thyroidectomies are commonly performed in cases of Thy-3A, Thy- $3 \mathrm{~F}$ and Thy-4 lesions, which are either indeterminate or show features of follicular or malignant disease.

\section{Objectives}

The study aimed to compare pre-operative cytology results classified as Thy-3 (indeterminate) or Thy-4 (suggestive of malignancy) against post-operative histology, in order to establish the malignancy rates of 'indeterminate' lesions, and correlate this with features on ultrasonography.

\section{Methods}

A retrospective review was conducted of the operative histology and ultrasound grading of the last 50 hemi-thyroidectomies performed for Thy-3 or Thy-4 FNAC lesions at the Norfolk and Norwich Hospital.

\section{Results}

Thirty-three patients (66 per cent) with a Thy- 3 or Thy- 4 cytological grading had benign disease post-operatively compared to 17 patients ( 34 per cent) that were malignant. Of the 36 lesions graded Thy-F (suggestive of follicular malignancy), 22 were benign, whilst 14 (39 per cent) were malignant, which is a higher rate than that reported in some published studies. Patients with Thy-A grading (atypical features) showed lower malignancy rates, affecting one out of nine patients ( 11 per cent). Three patients had Thy-4 FNAC (suggestive of malignancy) pre-operatively, yet two out of three ( 66 per cent) ended up having benign disease.

\section{Conclusion}

Our data support the use of hemi-thyroidectomy in the investigation of Thy- $3 \mathrm{~F}$ lesions. The high malignancy rate (34 per cent) for this group in our study demonstrates the importance of obtaining a histological diagnosis for these lesions.

An analysis of true- and false-positive results of vocal fold uptake in positron emission tomography/computed tomography imaging

\section{N Seymour, G Burkill, M Harries}

From the Brighton and Sussex University Hospital NHS Trust, Brighton

\section{Introduction}

Positron emission tomography/computed tomography (PET/CT) with fluorine-18 fluorodeoxy-D-glucose $(18 \mathrm{~F}-$ FDG) has a major role in the investigation of head and neck cancers. The $18 \mathrm{~F}-\mathrm{FDG}$ is not a tumour-specific tracer and can accumulate in benign pathology. Therefore
PET/CT scan interpretation difficulties are common in the head and neck, which can produce false-positive results.

\section{Objective}

This study aimed to investigate patients detected as having abnormal vocal fold uptake on 18 F-FDG PET/CT.

\section{Methods}

The PET/CT scans were identified over a 15-month period where reports contained evidence of unilateral vocal fold uptake or vocal fold pathology. Patient notes and laryngoscopy results were analysed.

\section{Results}

Forty-six patients were identified as having abnormal vocal fold uptake on PET/CT. Twenty-three patients underwent PET/CT and flexible laryngoscopy: 61 per cent of patients had true-positive PET/CT scan results and 39 per cent had false-positive scan results.

\section{Conclusion}

The majority of patients referred to ENT because of abnormal findings on PET/CT scans had true-positive findings. Asymmetric 18 F-FDG uptake should raise suspicion of vocal fold pathology, accepting a false-positive rate of approximately 40 per cent.

\section{Health gains and cost-utility analysis for septorhinoplasty}

\section{Oladokun, A Baumgart, I Baumann, O C Bulut}

From the Mannheim Institute of Public Health and University Hospital Heidelberg, Germany

\section{Background}

Demand for septorhinoplasty is rising internationally. Associated health benefits and the cost at which these are acquired remain controversial. This study aimed to assess health gains associated with septorhinoplasty and to perform a cost-utility analysis for the procedure.

\section{Methods}

A total of 103 patients undergoing septorhinoplasty at a German tertiary-level hospital completed the Short Form36 ('SF-36') and satisfaction questionnaires before and 12 months after their procedure. The Short Form 6D ('SF6D') instrument was used to determine quality-adjusted life year values from Short Form-36 responses, thus allowing estimation of pre- and post-operative health utilities. Health utility gains after septorhinoplasty were combined with cost data to estimate procedural costs per quality-adjusted life years gained (cost-utility) from the statutory health insurer's perspective.

\section{Results}

Sixty-nine patients completed all questionnaires pre-operatively and one-year post-operatively. Reported qualityadjusted life year values were 0.70 pre-operatively and 0.74 one-year post-operatively, indicating health gains of 0.04 quality-adjusted life years ( 95 per cent confidence interval $=$ $0.01-0.07)$. Patients satisfied with their procedures had significant health utility gains, whilst dissatisfied patients did not experience any significant gains. The cost of septorhinoplasty to the statutory health insurer in this study was $€ 3487.69$. When compared to the baseline, the incremental utility ratio for septorhinoplasty was €94 797.30 per quality-adjusted life year gained. Sensitivity analysis, based 
on variations in outcomes and reimbursement rates, yielded a best-case incremental utility ratio of $€ 50030.91$ and a worstcase incremental utility ratio of $€ 516762.86$.

\section{Conclusion}

This study successfully estimated health utilities, health gains and cost-utility for septorhinoplasty, and is the first study to do so. The findings indicate that septorhinoplasty has associated health gains, but these are acquired at a high cost-utility ratio.

Adjustable flange tube in surgical tracheostomy: audit of practice

S M Mummadi, C Czyrko, A Darr

From the University Hospitals of North Midlands, Stoke-on-Trent

\section{Introduction}

The National Confidential Enquiry into Patient Outcome and Death report 'On the Right Trach?' (published in 2014) highlighted some key concerns regarding neck stomas in obese patients. Obese patients were more likely to undergo a tracheostomy and were at a higher risk of tracheostomy-related complications. Fifty per cent of unplanned tube changes occurred in patients with a body mass index of more than $30 \mathrm{~kg} / \mathrm{m}^{2}$. Despite this, knowledge surrounding the appropriate use of adjustable flange tracheostomy tubes in this cohort of patients is poor, as evidenced by their use in only 18.8 per cent of obese patients.

\section{Objective}

The study aimed to assess documentation for neck assessment, thyroid isthmus control, tracheal incision type, tube type (single $v s$ double lumen), tube length and flange position in patients who had an adjustable flange tracheostomy tube inserted. These variables are based on best practice guidance provided in the aforementioned National Confidential Enquiry into Patient Outcome and Death report.

\section{Methods}

A retrospective review was conducted of operation records and case notes for all adjustable flange tracheostomies performed over a three-month period at a tertiary trauma centre.

Results

Adjustable flange tubes were utilised in 11 per cent of all tracheostomies performed. The documentation for assessment was frequently incomplete, with neck assessment being the most frequently omitted variable. Documentation completion and adjustable flange tube uptake were poorer in cases of tracheostomies performed by non-ENT specialists.

\section{Conclusion}

There is significant scope for improvement in the documentation for assessment when using adjustable flange tubes.
Further education on the availability of this tube type may improve uptake in obese patients. Education, particularly in non-ENT specialties, may also improve the documentation for key areas of assessment that may help reduce the rate of tracheostomy-related complications.

Doctor Google will see you now: an assessment of quality and readability of web-based information on cochlear implants, aimed at patients

N Seymour ${ }^{1}$, R Lakhani ${ }^{2}$, B Hartley ${ }^{2}$, L Cochrane ${ }^{2}$, C Jephson ${ }^{2}$

From the ${ }^{1}$ Queen's Medical Centre, Nottingham University Hospital Trust and ${ }^{2}$ Great Ormond Street Hospital, London

\section{Introduction}

Shared decision-making between patients and doctors is an integral part of modern healthcare. Cochlear implant candidates and their families tend to be motivated, and they often seek information from sources such as the internet, which lends itself as a powerful tool in providing healthrelated information for such conditions.

\section{Objective}

Patients should have access to high-quality health information websites on which they can base their decisionmaking. We aimed to objectively measure website quality related to cochlear implantation.

\section{Methods}

Selected patient information websites were scored, depending on how highly they were ranked on search engines and if they were ranked on more than one of the search engines used. The top 40 websites from 3 major search engines were analysed. The quality of each website was scored using the Discern tool, and readability was scored using the Flesch-Kincaid Reading Ease test and the Gunning Fog Index.

\section{Results}

The average Flesch-Kincaid score was 49.7, indicating that the material can be understood by most $15-17$ year olds. The average Gunning Fog Index was 13.1, which equals the reading age of an 18 year old.

\section{Conclusion}

Internet-based information on cochlear implantation is of varied quality, and it is written above the expected reading level of a 13 year old in the UK. The average patient may not benefit from this information, given difficulties in readability. Despite these limitations, there are a wide range of online resources on cochlear implantation, and the top 10 websites outlined by the study may be used as a suggested reading list for cochlear implant candidates. 